\title{
The aftershocks of the LACC earthquake have begun: brace yourselves
}

\section{Correspondence to}

Dr Mario M Leitao Jr, Memorial Sloan-Kettering Cancer Center, New York City, NY 10065, USA; leitaom@mskcc.org

Received 10 December 2019 Accepted 11 December 2019 Published Online First 9 January 2020

\section{SLinked}

- http://dx.doi.org/10.1136/ ijgc-2019-000838

\section{Check for updates}

(C) IGCS and ESG0 2020. № commercial re-use. See rights and permissions. Published by BMJ.

To cite: Leitao Jr MM. Int J Gynecol Cancer 2020;30:157159.

Mario M Leitao Jr

Randomized controlled trials have shown that laparoscopy, in comparison to laparotomy, results in lower peri-operative complication rates and improved quality of life in women undergoing surgery for newly diagnosed endometrial cancer. ${ }^{1-5}$ Furthermore, two large randomized controlled trials have shown that oncologic outcomes, such as progression-free survival, recurrence-free survival, and overall survival, are not compromised with the use of laparoscopy in this patient population. ${ }^{34}$

The Gynecologic Oncology Group (GOG) LAP2 study did not meet the protocol-specified criteria for concluding that laparoscopy was non-inferior to laparotomy based on recurrence rates. ${ }^{3}$ The 3 -year estimated cumulative incidence of recurrence in both the laparoscopy and laparotomy cohorts was less than expected at $11.4 \%$ and $10.2 \%$, respectively. ${ }^{3}$ The 5 -year overall survival rates were $90 \%$ for both groups. With more than 2500 patients enrolled, it likely does not matter that statistics-based criteria were not met in a 'real-world' clinical sense. The cumulative incidence of recurrence and overall survival curves were superimposed, telling us we can realistically and sensibly conclude there is no compromise to oncologic outcomes despite the statistical issue. The Laparoscopic Approach to Cancer of the Endometrium (LACE) randomized trial subsequently confirmed the outcome similarities of laparoscopy and laparotomy, with 4.5-year disease-free survival rates of $81 \%$ and overall survival rates of $92 \%$ in both cohorts. ${ }^{4}$

The nationwide adoption of laparoscopy for the management of endometrial cancer has had significant positive impacts. Data from the American College of Surgeons National Quality Improvement Programme (ACS-NSQIP) confirmed the randomized trial data while reporting complication rates of $31 \%$ after laparotomy compared with $12 \%$ after minimally invasive surgery $(p<0.0001)$ among 2076 cases included in the analysis. ${ }^{6}$ Cost modeling also demonstrated a savings of $\$ 2.8$ million in the laparoscopic cohort, attributable to hospital stay and reductions in complications. ${ }^{6}$ The authors suggested a $\$ 534$ million savings could be realized with the use of minimally invasive surgery in $90 \%$ of all patients with endometrial cancer in the USA, with a simultaneous decrease in the use of laparotomy. A more recent analysis of the
ACS-NSQIP database with 12283 patients reported an increase in minimally invasive surgery rates from $24 \%$ in 2008 to $71 \%$ in 2014 ( $p<0.001)$, which led to a $40 \%$ reduction in overall 30 -day complications and a 2-day reduction in median length of hospital stay. ${ }^{7}$ These data likely underestimate the benefits of minimally invasive surgery since they do not take into account the serious medical and economic impact of the late complications of laparotomy, such as incisional herniae and adhesive bowel disease.

There are substantial prospective and observational data to fully support minimally invasive surgery as the standard surgical approach in women with endometrial cancer. However, cervical cancer is a different disease, and the safety of minimally invasive surgery has been contested with the findings of the randomized Laparoscopic Approach to Cervical Cancer (LACC) trial. ${ }^{8}$ The findings of the study showed an inferior disease-free survival and overall survival in patients who underwent minimally invasive surgery compared with laparotomy. The debate over these results continues, and new trials to address the issue are in development or have been opened and are accruing. ${ }^{9}$ Unfortunately, the LACC quake has pushed aftermath tremors into the management of other malignancies. The current study, likely the first of many retrospective observational studies in this area, has now 're-assessed' the use of minimally invasive surgery in endometrial cancer.

Song and colleagues from The Ottawa Hospital in Canada report on a retrospective analysis of 179 stage I 'intermediate-risk' patients with endometrial cancer treated over a 6 -year period. ${ }^{10}$ The authors report an increased risk of recurrence with the use of the robotic platform over laparotomy, but with no overall survival difference. Minimally invasive surgery in cancer surgery is obviously a hot topic in our field right now; just a few years ago this paper would not have been a lead article and would not have warranted an accompanying editorial. But such are the times, when a retrospective series reporting inferior outcomes with minimally invasive surgery is a lead article and one reporting no difference is not accepted for publication due to the flaws of retrospective data analysis. 


\section{Editorial}

The authors refer to the LACC trial in the context of their findings, but again, the LACC trial was specific for patients with cervical cancer and not endometrial cancer, which is a distinct disease with unique management strategies. The authors indicate that GOG LAP2 and the LACE trial included mostly patients with 'low-risk' disease. The majority of patients likely had 'low-risk' disease; however, a significant number of patients had 'intermediate-risk' diseasesimilar to those in the Song study - or even higher-risk disease. In GOG LAP2, 20\% of the randomized cases were of non-endometrioid high-risk histology, with a total of 492 such cases. ${ }^{1}$ Additionally, $23 \%$ of randomized cases were International Federation of Gynecology and Obstetrics (FIG0) stage II or higher, with a total of 582 such cases. ${ }^{1}$ Some of the non-endometrioid cases were likely in the higher-stage cohort as well, but the point is that there were significantly more higher-risk cases in LAP2 than in the current series, accounting for at least $20 \%$ of the randomized cases. Furthermore, there were likely more 'intermediate-risk' cases in the randomized endometrioid stage I cases. This high number and proportion of more than 'low-risk' cases should have led to some separation of the recurrence-free survival and overall survival curves if minimally invasive surgery was truly leading to inferior outcomes, or one could say that not enough of such cases were in the overall cohort to have had any recognizable impact. Sub-group analyses in GOG LAP2 revealed there was no effect of minimally invasive surgery on the rate of recurrence for patients with $>50 \%$ myoinvasion $(n=594)$, serous histology ( $n=289)$, or stage IIIC disease $(n=226)$, as well as for other sub-groups. ${ }^{3}$ I realize that sub-group analyses are post hoc and also hypothesis-generating, but referring to the LAP2 sub-group analyses is appropriate considering the much smaller cohort of even lower-risk patients retrospectively analyzed in the current series.

The authors of the current study also make a point that GOG LAP2 and the LACE trial included only non-robotic laparoscopic approaches and seem to imply that the robotic platform is nonlaparoscopic. The robotic platform, however, is simply a tool with which to perform laparoscopy. It is a computer-enhanced surgical tool that overcomes some of the limitations of traditional laparoscopic instruments and has allowed for a significant increase in minimally invasive surgery rates, but it is still a laparoscopic instrument. Minimally invasive surgery-associated outcomes should be analyzed without differentiation of type of instrument used.

Assessing the full gamut of prospective and retrospective data is invaluable in helping us select the best treatments for our patients, while trying to find an acceptable balance between survival outcomes and quality of life. Accepting the findings of a single study as dogma is imprudent and ill advised. For each study, we should ask ourselves if the results make sense and if they apply to all patients. In the current study of only 179 cases there were no recurrences in the laparotomy cohort, which is concerning considering 'intermediate-risk' cases are associated with an approximately $4 \%$ risk of recurrence based on the study referenced by the authors. ${ }^{11}$ The fact that none of the patients in the laparotomy cohort recurred may be an anomaly, further confusing the analysis.

There are other key concerns with the current study. Of only eight total recurrences, all were in the robotic cohort, and the median follow-up times between the two cohorts varied (5.9 years in the robotic cohort compared with 4.2 years in the laparotomy cohort). This 19-month difference was not statistically significant, further highlighting the statistical limitation of a small series. Difference in follow-up time has always been a main criticism of retrospective series in cervical cancer even before the LACC trial. Differences in follow-up times may or may not be important. For example, if the median follow-up time is 12 months in one group and 29 months in the other group, and the median time to recurrence is 18 months with a range of 12-28 months, then the results are problematic. On the other hand, if the median follow-up time is 50 months in one group and 69 months in the other, and the median time to recurrence is 18 months and nearly all patients recur within 30 months, then this median follow-up time difference is irrelevant. In the current study, two (25\%) of the eight recurrences occurred at 5.2 and 5.4 years, which raises red flags considering the 4.2year median follow-up time of the laparotomy cohort. Furthermore, these were both distant recurrences to the lung. A distant recurrence more than 5 years after surgery brings into question biological and mechanistic factors rather than surgical approach. A third lung recurrence occurred 2.7 years after surgery. Again, relatively quick recurrences in the vagina, pelvis, peritoneum, or trocar sites due to subpar surgery is possible, but I struggle to understand the causal association with other sites of recurrence.

Another concern with the current series is that the median time to the initiation of adjuvant radiation therapy was 19 days (nearly 3 weeks) longer in the robotic cohort, and none of these patients started radiation before 46 days (1.5 months) of surgery compared with 22 days ( 0.7 months) in the laparotomy cohort. Interestingly, another two $(25 \%)$ of the eight recurrences occurred at 22 days and 15 days, well before any radiation was initiated in the robotic cohort. Randomized data have shown that radiation therapy does impact local recurrence risk but has no impact on overall survival. Would these recurrences have been avoided if radiation therapy had not been withheld? Of note, both recurrences were nodal. So was minimally invasive surgery the culprit of recurrence, or was it missed nodal disease? The mean (not median) nodal counts in both cohorts were less than 10, which some may consider inadequate. Outside the context of sentinel lymph node mapping, the more nodes removed the higher the likelihood of finding and removing nodal disease.

The inevitable aftershocks of the LACC earthquake have begun. Brace yourselves for the wave of incoming observational data assessing minimally invasive surgery and outcomes in higher-risk endometrial cancers. External validation of surgical randomized controlled trials is somewhat problematic, as surgery is not the objective equivalent of drug administration, with its limited variables, but a subjective process reliant on surgeon and institutional experience and acumen. This not only applies to the LACC trial but also to trials with reassuring and improved outcomes, such as those of GOG LAP2 and LACE. Results from surgical randomized controlled trials or from single-institution or multi-center retrospective analyses do not apply to all surgeons. Surgeons should keep close track of their data, especially with regard to survival outcomes, and compare their results to those of the literature. My question to Song and his colleagues is: will they stop using the robotic platform, or minimally invasive surgery in general, for this cohort of patients now? Will they also stop using minimally invasive surgery in 'high-risk' patients with endometrial cancer? This change may be appropriate for their institutional minimally invasive 
surgery program and practice; however, their findings cannot be generalized to the gynecologic oncology community as a whole.

Twitter Mario M Leitao Jr @leitaomd

Contributors $\mathrm{ML}$ is the sole author of this editorial.

Funding The author has not declared a specific grant for this research from any funding agency in the public, commercial or not-for-profit sectors.

Competing interests None declared.

Patient consent for publication Not required.

Provenance and peer review Commissioned; internally peer reviewed.

\section{REFERENCES}

1 Walker JL, Piedmonte MR, Spirtos NM, et al. Laparoscopy compared with laparotomy for comprehensive surgical staging of uterine cancer: Gynecologic Oncology Group study LAP2. J Clin Oncol 2009;27:5331-6.

2 Kornblith $A B$, Huang HQ, Walker JL, et al. Quality of life of patients with endometrial cancer undergoing laparoscopic International Federation of Gynecology and Obstetrics staging compared with laparotomy: a Gynecologic Oncology Group study. J Clin Oncol 2009;27:5337-42.

3 Walker JL, Piedmonte MR, Spirtos NM, et al. Recurrence and survival after random assignment to laparoscopy versus laparotomy for comprehensive surgical staging of uterine cancer: Gynecologic Oncology Group LAP2 study. J Clin Oncol 2012;30:695-700.

4 Janda M, Gebski V, Davies LC, et al. Effect of total laparoscopic hysterectomy vs total abdominal hysterectomy on diseasefree survival among women with stage I endometrial cancer: a randomized clinical trial. JAMA 2017;317:1224-33.

5 Janda M, Gebski V, Brand A, et al. Quality of life after total laparoscopic hysterectomy versus total abdominal hysterectomy for stage I endometrial cancer (LACE): a randomised trial. Lancet Oncol 2010;11:772-80.

6 Scalici J, Laughlin BB, Finan MA, et al. The trend towards minimally invasive surgery (MIS) for endometrial cancer: an ACS-NSQIP evaluation of surgical outcomes. Gynecol Oncol 2015;136:512-5.

7 Casarin J, Multinu F, Ubl DS, et al. Adoption of minimally invasive surgery and decrease in surgical morbidity for endometrial cancer treatment in the United States. Obstet Gynecol 2018;131:304-11.

8 Ramirez PT, Frumovitz M, Pareja R, et al. Minimally invasive versus abdominal radical hysterectomy for cervical cancer. $N$ Engl $\mathrm{J}$ Med 2018;379:1895-904.

9 Falconer H, Palsdottir K, Stalberg K, et al. Robot-assisted approach to cervical cancer (RACC): an international multi-center, open-label randomized controlled trial. Int J Gynecol Cancer 2019;29:1072-6.

10 Song J, Le T, Hopkins L, et al. A comparison of disease recurrence between robotic approach versus laparotomy approach in patients with intermediate-risk endometrial. Int J Gynecol Cancer 2020;30:160-6.

11 Creutzberg CL, van Putten WLJ, Koper PCM, et al. Surgery and postoperative radiotherapy versus surgery alone for patients with stage- 1 endometrial carcinoma: multicentre randomised trial. The Lancet 2000;355:1404-11. 